\title{
Enhanced Drainage Protocol in Large Amoebic Liver Abscess
}

\author{
Jignesh A. Gandhi, MS, DNB, FMAS, FIAGES, FALS, DLS (France), Robotic Surgery (USA) ${ }^{10}$ \\ Pravin H. Shinde, MBBS, DNB, FIAGES ${ }^{2}{ }^{-}$Sadashiv N. Chaudhari, MBBS, DNB ${ }^{2}$ \\ Amay M. Banker, MBBS , MS²®
}

${ }^{1}$ Department of Surgery, Global Hospital Mumbai, Parel, Mumbai, India
${ }^{2}$ Department of General Surgery, Seth G.S. Medical College \& KEM
Hospital, Mumbai, India

Surg J (NY) 2021;7:e351-e356.

\author{
Address for correspondence Pravin H. Shinde, MBBS, DNB, FIAGES, \\ Assistant Professor, Department of General Surgery, Seth G.S. Medical \\ College \& KEM Hospital Parel, Mumbai 400012, India \\ (e-mail: pravinshinde@kem.edu).
}

\begin{abstract}
Keywords

- amoebic liver abscess

- drain

- pigtail

- biliary communication

Background Amebic liver abscess (ALA) contributes significantly to morbidity and mortality in patients of the developing world. Even though medical management is the primary modality of treatment, $15 \%$ of the cases are refractory and require intervention for drainage. Pigtail catheterization is inefficient and results in a long duration of hospital stay. So, we conducted a prospective observational study to determine the efficacy and safety of drainage of large ALA using a wide bore 24 French (Fr) drain compared with a conventionally used $10 \mathrm{Fr}$ pigtail catheter.

Materials and Methods A single center prospective observational study was conducted over a period of 5 years and data of 122 patients was collected. After starting empirical medical therapy, patients underwent drainage of ALA with either a 10 French pigtail or a 24 Fr drain. The primary outcome variables were resolution of clinical symptoms such as fever and pain in abdomen, length of hospital stay, and resolution of abscess on imaging at day 3 . Secondary outcome was complications related to the procedures.

Results Data of 122 patients was collected. Males constituted a vast majority (96\%) of the study population and the fifth decade was the most common age group involved. Alcoholics had a higher chance of developing a large ALA. Sixty-eight patients underwent drainage of the ALA using a $24 \mathrm{Fr}$ drain which resulted in faster resolution of symptoms (2.4 vs. 5.1 days, $p$-value 0.033 ), a shorter duration of catheter in situ (6.4 vs. $13.2, p$-value 0.011 ), and a faster drainage of ALA (residual volume at day $3 ; 177$ vs. $212 \mathrm{~mL}, p$-value 0.021 ). Twenty-eight patients had a biliary communication of which 26 required therapeutic endoscopic retrograde cholangiopancreatography.

Conclusion In patients with a large ALA, placement of a wide bore $24 \mathrm{Fr}$ catheter hastens recovery of the patients when compared with drainage with a standard $10 \mathrm{Fr}$ pigtail catheter. Placement of a biliary stent serves as a useful adjunct for their management and it may obliviate the need for a major biliary diversion surgery.
\end{abstract}

Amoebiasis is a common parasitic infection affecting approximately 50 million people worldwide and resulting in approximately 100,000 deaths per year. ${ }^{1-3}$ Ninety percent

received

January 15, 2021

accepted after revision

October 26, 2021
DOI https://doi.org/

10.1055/s-0041-1740625.

ISSN 2378-5128. of such patients remain asymptomatic and the liver is affected in 3 to $9 \%$ of cases. This makes amebic liver abscess (ALA) the most common extraintestinal manifestation of
(C) 2021. The Author(s).

This is an open access article published by Thieme under the terms of the Creative Commons Attribution License, permitting unrestricted use, distribution, and reproduction so long as the original work is properly cited. (https://creativecommons.org/licenses/by/4.0/)

Thieme Medical Publishers, Inc., 333 Seventh Avenue, 18th Floor, New York, NY 10001, USA 
amebiasis. ${ }^{4}$ The mainstay of treatment for ALA is medical, but up to $15 \%$ of the cases may be refractory to medical management. ${ }^{5}$ Presence of communication with the biliary tree is considered one of the reasons for non-resolution of ALA. ${ }^{4,6}$ Imaging-guided percutaneous placement of a catheter has shown to be superior to needle aspiration for such refractory ALAs. ${ }^{6-11}$ However, in our experience we found that the pigtail catheter becomes nonfunctional due to repeated blockage and requires repeated flushing which can introduce secondary infection ultimately resulting in a longer hospital stay. So, we conducted a prospective observational study to determine the efficacy and safety of drainage of large ALA using a wide bore 24 French (Fr) drain compared with a conventionally used $10 \mathrm{Fr}$ pigtail catheter. We came across 28 cases of a biliary communication with the ALA in the past 5 years. In this study, we also present our experience in the management of such cases with the use of therapeutic endoscopic retrograde cholangiopancreatography (ERCP).

\section{Methodology}

This was a single center prospective observational study conducted over a period of 5 years between October 2014 and June 2019 after approval from the institutional ethics committee. The amoebic etiology was suspected by the ultrasonographic (US) or computed tomographic (CT) appearance of the lesion and the diagnosis was confirmed by an enzyme-linked immunosorbent assay (ELISA). ${ }^{12}$ Abscess cavities larger than $3 \mathrm{~cm}$ were considered large abscess for the purpose of this study. Patients with abscess cavities smaller than $3 \mathrm{~cm}$ in their greatest dimension; ruptured liver abscess; concomitant biliary tract malignancy; and uncorrectable coagulopathy were excluded from the study. A total of 122 patients were enrolled in the present cohort and were worked up in terms of detailed history and clinical examination. Laboratory and radiological investigations included a complete hemogram, liver function tests (LFTs), coagulation profile including prothrombin time and international normalized ratio, a US and a contrast enhanced CT scan of the abdomen.

After informed consent, all patients were started on intravenous metronidazole (500 mg every 8 hourly). Coagulopathy was corrected prior to any intervention. The modalities used along with medical therapy were insertion of a $10 \mathrm{Fr}$ pigtail catheter and insertion of a $24 \mathrm{Fr}$ drain with a blunt tipped trocar.

A 10 Fr pigtail catheter was used ALA showing an impending rupture on US ( $\mathrm{rim}<10 \mathrm{~mm}$ ), for left lobe abscess, and patients with abscess volume $<200 \mathrm{~mL}$ and maximum diameter $<4 \mathrm{~cm}$ on US. This was performed under USG guidance with all aseptic precautions.

A $24 \mathrm{Fr}$ catheter insertion was performed for peripheral abscesses, abscesses with partially or uniquified ALA with a favorable anatomy on CT scan, and abscesses with maximum diameter $>4 \mathrm{~cm}$ on US. Using a No.11 blade a small stab was made on the anaesthetized skin. Under US guidance, a 24 French drain with a blunt tipped trocar was then introduced into the abscess cavity, a technique similar to that described for trocar thoracostomy. ${ }^{13}$

The aspirate was sent for routine microscopy and culture sensitivity and the antibiotics were adjusted accordingly. Patients with negative culture results were continued on the initial drug treatment for 14 days. Daily drain output was monitored for the presence of bilious contamination. Bile in drain was suggestive of a biliary fistula and those patients who had a consistent biliary output of $>200 \mathrm{~mL} / \mathrm{d}$ for $>7$ days were referred for an ERCP. After obtaining a cholangiogram, therapeutic ERCP was done which included a sphincterotomy followed by the placement of a $10-\mathrm{cm} 7 \mathrm{Fr}$ plastic pigtail stent up to the site of fistula wherever possible. Follow-up US was done on day 3 and all patients had a routine follow-up at 6 weeks.

The percutaneous drains were removed when drain output was $<20 \mathrm{~mL} / \mathrm{d}$ for 2 consecutive days or the maximum diameter of the abscess was $<3 \mathrm{~cm}$ on follow-up US. Clinically stable patients with complete resolution of symptoms were discharged with pigtail catheter in situ with follow-up and removal on outpatient department (OPD) basis. Patients with a 24 Fr drain were discharged only after removal of drain. The biliary stent was removed after 6 weeks. The primary outcome variables were resolution of clinical symptoms such as fever and pain in abdomen, length of hospital stay, and resolution of abscess on imaging at day 3 . Secondary outcome was complications related to the procedures. Chisquare test was used for the analysis of categorical variables and a logistic regression model was developed for continuous variables. Data was analyzed using SPSS 26.0 (Statistical Package for Social Sciences; IBM, Chicago, IL).

\section{Results}

\section{Demographics}

The present cohort consisted of 122 patients diagnosed with ALA. Males constituted a majority of the study population (96\%). The average age of the cohort was 42 years. Ninetythree (76\%) patients had a history of chronic alcohol consumption and diabetes (56\%) was the most common comorbidity seen (-Table $\mathbf{1}$ ).

\section{Investigations}

A total of 101 (82\%) patients had leukocytosis on admission. Twenty-four (18\%) patients had elevated bilirubin levels. Incidentally all these patients had a biliary communication with the liver abscess. Cultures were found to be positive in 18 of $122(14.7 \%)$ of the cases. Among the pus culture positive cases Escherichia coli was isolated most frequently (8 of 18 culture positive patients).

It was closely followed by Klebsiella spp. which was isolated in six cases. The rest were sterile abscesses (-Table 2). Out of the 122 patients, abscess was seen in the right lobe in 92 cases. Twenty-two cases were of left lobe abscesses while eight cases had abscesses involving both the lobes (-Fig. 1). The mean volume of the ALA was $656 \mathrm{~mL}$ (range $320-1,300 \mathrm{~mL}$ ) on US corresponding to an average size of $4.2 \mathrm{~cm}$ (range $2.2-9.6 \mathrm{~cm}$ ) in the largest diameter. 
Table 1 Patient demographics

\begin{tabular}{|l|l|}
\hline Total patients & 122 \\
\hline Sex & \\
\hline Male & $117(96 \%)$ \\
\hline Female & $5(4 \%)$ \\
\hline Age, years & $42(35-56)$ \\
\hline Chronic alcohol use $^{\text {COMORBIDITIES }}{ }^{\text {a }}$ & $93(76.2 \%)$ \\
\hline Diabetes mellitus & \\
\hline Anemia & $69(56 \%)$ \\
\hline Hypertension & $54(44 \%)$ \\
\hline COPD & $48(39 \%)$ \\
\hline Ischemic heart disease & $14(11 \%)$ \\
\hline
\end{tabular}

Abbreviation: COPD, chronic obstructive pulmonary disease. Note: Values are listed as either number (percentage), mean ( \pm standard deviation).

${ }^{\mathrm{a}}$ Comorbidities coexisted in a few patients.

Table 2 investigations

\begin{tabular}{|l|l|}
\hline Total patients & 122 \\
\hline TLC counts/mm ${ }^{3}$ & $18,2334( \pm 3,319)$ \\
\hline Hemoglobin, mg/dL & $11.5( \pm 2.14)$ \\
\hline ALT, U/L & $47.78( \pm 16.66)$ \\
\hline AST, U/L & $31.33( \pm 12.78)$ \\
\hline ALP, U/L & $196( \pm 76.98)$ \\
\hline Total bilirubin, $\mathrm{mg} / \mathrm{dL}$ & $1.29( \pm 0.44)$ \\
\hline US findings & \\
\hline Size, cm & $4.2( \pm 1.8)$ \\
\hline Volume, $\mathrm{mL}$ & $656( \pm 231)$ \\
\hline Pus culture & \\
\hline No growth & $104(85 \%)$ \\
\hline E. coli & $8(6 \%)$ \\
\hline Klebsiella species & $6(5 \%)$ \\
\hline Pseudomonas species & $2(2 \%)$ \\
\hline Acinetobacter & $2(2 \%)$ \\
\hline
\end{tabular}

Abbreviations: ALP, alkaline phosphatase; ALT, alanine transaminase; AST, aspartate transaminase; US, ultrasonography.

Note: Values are listed as mean (standard deviation) or number (percentage).

\section{Management}

Sixty-eight (55.7\%) patients underwent drainage with a $24 \mathrm{Fr}$ catheter. The average volume of the ALA was $977 \mathrm{~mL}(622-$ $1,300 \mathrm{~cm}$ ). Mean residual volume on day 3 in this group was $177 \mathrm{~mL}$ with an average duration of catheter in situ being 6.4 days (range 3-22 days). Complete resolution of clinical symptoms was early (average 2.4 days). The mean length of hospital stay was 7.1 days (range $4-24$ days) in this cohort.

\section{Anatomic Distribution of ALA}

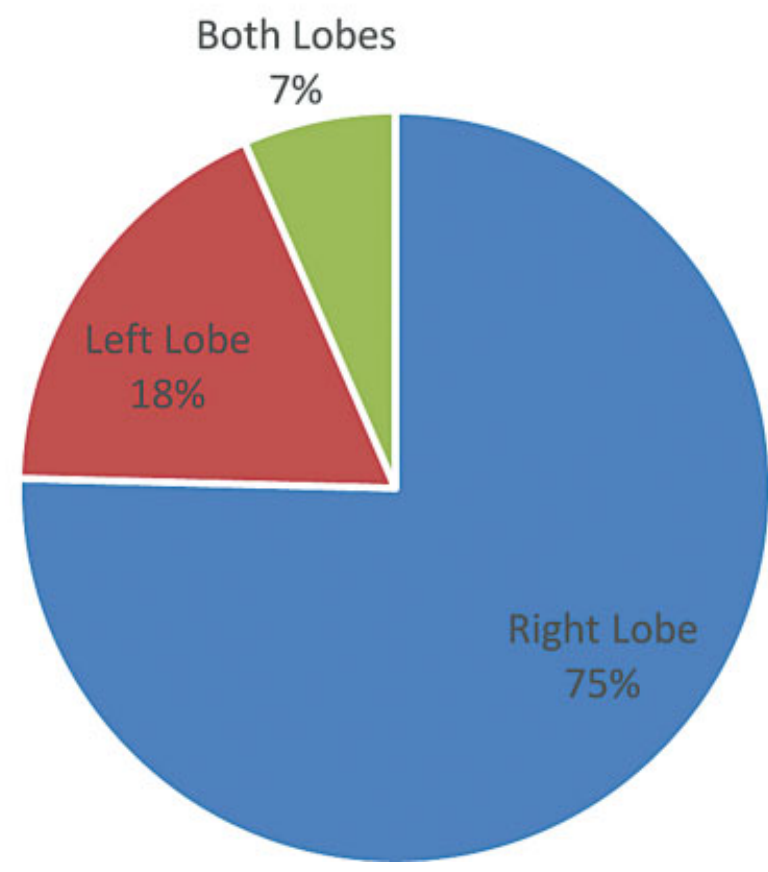

Fig. 1 Anatomical distribution of liver abscess.

Twelve patients complained of pain at insertion site which was managed with oral analgesics. There was one case of hemorrhage within the ALA which was managed conservatively. One case of catheter migration required repositioning and there was one incidence of pneumothorax which was managed by intercostal tube insertion ( - Table 3 ).

Insertion of a $10 \mathrm{Fr}$ pigtail was performed in 54 (44.3\%) cases. The mean volume of the ALA was $543 \mathrm{~mL}$ in this group. Repeat US on day 3 showed a mean residual volume of $212 \mathrm{~mL}$. The average duration of catheter in situ was longer (mean 13.2 days, range 6-24 days) and mean length of hospital stay was 7.3 days (4-18 days). The time taken for complete resolution of symptoms was 5.1 days. Accidental removal of pigtail was seen in six cases. A blocked pigtail was seen in $25 \%$ of the cases which required flushing.

\section{Statistical Analysis}

Both the groups were homogenous in terms of age and sex distribution. In our study the average size and volume of the ALA in the $24 \mathrm{Fr}$ catheter group was significantly higher (977 vs. $543 \mathrm{~mL}, p$-value 0.046). Despite the larger average size of the abscess, there was a significantly faster resolution of symptoms seen in the larger $24 \mathrm{Fr}$ drainage (2.4 vs. 5.1 days, $p$-value 0.033 ). The mean volume of the abscess on day 3 of drainage was also significantly lower in the group drained by the larger $24 \mathrm{Fr}$ drain (177 vs. $212 \mathrm{~mL}, p$-value 0.021 ). The average duration of drain in situ was also lower in the group drained by $24 \mathrm{Fr}$ drain (6.4 vs. 13.2 days, $p$-value 0.011 ) ( - Table 3 ). In three patients, small $(2-3 \mathrm{~cm})$ residual cavities were still present at 6 weeks after drainage; these patients were lost to further follow-up. There was no statistical 
Table 3 Comparison of $24 \mathrm{Fr}$ catheter vs. $10 \mathrm{Fr}$ pigtail

\begin{tabular}{|l|l|l|l|}
\hline & 24 Fr catheter $(\boldsymbol{n}=68)$ & 10 Frr pigtail $(\boldsymbol{n}=\mathbf{5 4})$ & $p$-Value \\
\hline Age & 41 & 44 & 0.988 \\
\hline Male sex & $64(94 \%)$ & $53(98 \%)$ & 0.876 \\
\hline Volume, $\mathrm{mL}$ & $977( \pm 344)$ & $543( \pm 268)$ & 0.046 \\
\hline Residual volume at day 3, $\mathrm{mL}$ & $177( \pm 81)$ & $212( \pm 94)$ & 0.021 \\
\hline Duration of catheter, days & $6.4( \pm 2.2)$ & $13.2( \pm 4.2)$ & 0.011 \\
\hline Resolution of symptoms, days & $2.4( \pm 1.2)$ & $5.1( \pm 3.7)$ & 0.033 \\
\hline Length of hospital stay, days & $7.1( \pm 3.7)$ & $7.3( \pm 2.9)$ & 0.512 \\
\hline Biliary communication & $20(29 \%)$ & $8(14 \%)$ & 0.233 \\
\hline Complications & & & \\
\hline Pain & 12 & 4 & - \\
\hline Hemorrhage & 1 & 0 & - \\
\hline Pneumothorax & 1 & 0 & - \\
\hline Migration/removal & 1 & 6 & - \\
\hline Block & 0 & 14 & - \\
\hline
\end{tabular}

Note: Continuous variables are listed as mean (standard deviation) and categorical variables as count (percentage).

difference between the two groups in terms of complete radiological resolution of the ALA.

\section{Biliary Communication}

Biliary communication was noted in 28 cases. This subset of patients had a longer duration of hospital stay (mean 16.7 days) and they took longer for complete resolution of symptoms (mean 9.3 days). Elevated serum bilirubin levels (mean $4.3 \mathrm{mg} / \mathrm{dL}$, range $2.2-9.9 \mathrm{mg} / \mathrm{dL}$ ) and alkaline phosphatase levels (mean $344 \mathrm{IU} / \mathrm{L}$, range 172-677 IU/L) were only seen in patients with a biliary communication. Amongst the patients with biliary communication, none had a left lobe abscess. The lesions with biliary communication tended to be larger with a mean volume of $990 \mathrm{~mL}$ (range $677-1,300 \mathrm{~mL}$ ). Twenty of the 28 cases were those requiring a larger $24 \mathrm{Fr}$ drain insertion. There was a spontaneous closure of the fistula in two cases. The remaining 26 cases were subjected to a therapeutic ERCP. No ERCP-related complications were noted in this cohort. Stenting led to the closure of biliary communication in all cases and no mortality was noted ( - Table 4 ).

\section{Discussion}

Liver abscess is a major disease of the gastrointestinal system seen in the tropics. ${ }^{14}$ ALA is a dead end for the trophozoite form of Entamoeba histolytica as there is no multiplication of the trophozoite form in the liver. The trophozoite survives on the hepatocytes leaving the connective tissue and the reticuloendothelial system intact. While metronidazole has remained the cornerstone of management of ALA since its acceptance, various studies have demonstrated early recovery with drainage of the abscess. ${ }^{6,8,9,15,16}$ In the later stages ALA usually consists of acellular proteinaceous debris, and a brown fluid which is likened to "Anchovy Paste" consisting of necrotic hepatocytes. A $10 \mathrm{Fr}$ pigtail catheter becomes nonfunctional due to blockage which may require repeated flushing which in turn can introduce secondary infections. This prompted us to drain the ALA with a wide bore $24 \mathrm{Fr}$ catheter to achieve more efficient drainage. In a similar study by Gadahire and Shrotriya, they demonstrated earlier recovery of ALA when drained by a 20 Fr catheter compared with a 10 Fr pigtail. ${ }^{9}$

Table 4 Comparison of ala with and without biliary communication

\begin{tabular}{|l|l|l|}
\hline & $\begin{array}{l}\text { With biliary } \\
\text { communication }(\boldsymbol{N}=\mathbf{2 8})\end{array}$ & $\begin{array}{l}\text { Without biliary } \\
\text { communication }(\boldsymbol{N}=\mathbf{9 4})\end{array}$ \\
\hline Volume, $\mathrm{mL}$ & $990( \pm 178)$ & $491( \pm 228)$ \\
\hline Serum bilirubin, $\mathrm{mg} / \mathrm{dL}$ & $4.3( \pm 1.1)$ & $0.8( \pm 0.21)$ \\
\hline Alkaline phosphatase, u/L & $344( \pm 158)$ & $167( \pm 49)$ \\
\hline Duration of catheter, days & $15.9( \pm 2.2)$ & $8.6( \pm 4.2)$ \\
\hline Resolution of symptoms, days & $9.3( \pm 2.8)$ & $4.1( \pm 1.7)$ \\
\hline Length of hospital stay, days & $16.7( \pm 3.6)$ & $6.9( \pm 3.3)$ \\
\hline
\end{tabular}

Note: Continuous variables are listed as mean (standard deviation) and categorical variables as count (percentage). 
The findings of our study demonstrate the superiority of a wide bore catheter drainage to conventional pigtail drainage in large ALA. Patients with large ALA who were drained with a $24 \mathrm{Fr}$ catheter had faster resolution of symptoms and had a lower mean residual abscess volume on day 3 . These findings are similar to those reported by Kumar and Singh. ${ }^{17}$ The wide bore of the catheter allowed for a faster drainage of the abscess which explains earlier recovery in our patients. While earlier studies have shown a decrease in the length of hospital stay, no statistical difference was found in the present study. ${ }^{9,17}$ In both these series, patients were discharged with the catheter in situ while no patient in the present study was discharged with the $24 \mathrm{Fr}$ catheter in situ in this study.

Complications were noted in $25 \%$ of the cases of the catheter group with pain at the site of insertion being the most common (17\%). All these cases were managed with analgesics. A pneumothorax was noted in one case which was managed by the insertion of the thoracic tube. The patient had an uneventful recovery and was discharged on postoperative day 16. One patient developed hemorrhage into the abscess cavity which was managed conservatively. While pain was less in the pigtail group, accidental removal of the pigtail was seen in six patients. All these patients required a repeat intervention. Twenty-five percent of the patients complained of a blocked pigtail requiring repeated flushing and resulting in a longer mean duration of pigtail in situ.

ALA is found to be 3 to 10 times more common in males. ${ }^{18}$ In this study, the males constituted $96 \%$ of the study population. The fifth decade was the most affected age group. The clinical presentation of our patients was similar to that described in previous reports. ${ }^{11,18,19}$ Seventy-six of the patients had a history of chronic alcohol use in the present study. Duration of alcohol use has been shown to be a risk factor for the development of ALA. ${ }^{20}$ It was seen in animal studies that increased iron deposition increases the invasiveness of E. histolytica. Makkar et al hypothesized that since chronic alcoholism increases iron deposition in liver, it would explain the increased risk of ALA in chronic alcoholics. $^{21}$ A majority of our patients also presented with leukocytosis. Presentation with jaundice proved to be a useful clinical marker of abscess erosion into the biliary tract. Varying incidence $(23-27 \%)$ of jaundice has been reported in patients with ALA. ${ }^{6,22,23}$ While many pathogenetic mechanisms have been suggested, obstruction of the biliary system by the abscess is most widely accepted. 6,24 Nigam et al showed that multiple, large abscesses, have been directly related to the elevation of serum bilirubin levels. ${ }^{23}$ Another possible mechanism is that large ALA compresses the biliary radicles producing jaundice and finally penetrates the tough fibrous vasculobiliary sheath which surrounds the portal triad structures with resultant biliary communication. ${ }^{6}$ All our patients with jaundice showed a rapid decline in serum bilirubin to normal levels after drainage of the ALA, again establishing obstruction as a cause of jaundice in these patients. Pus cultures were negative in $85 \%$ of the cases and Escherichia coli and Klebsiella were the most commonly isolated organisms. This result is similar to the published literature. $^{11,25,26}$

The incidence of biliary communication was $22 \%$ in the present study which is similar to that reported in existing literature. $^{4,6,16}$ The presence of biliary communication was associated with the need of a longer duration of hospital stay. These patients also had a larger abscess, were more frequently associated with jaundice, and required catheter drainage for a longer duration. Agarwal et al also presented findings similar to these. ${ }^{6}$ In two patients there was a spontaneous closure of the biliary fistula probably due to the tamponading effect of the hepatic parenchyma and low pressure of the now unobstructed biliary system after drainage of the ALA as suggested by Do et al. ${ }^{27}$ The remaining cases required a therapeutic ERCP with sphincterotomy and placement of a stent for resolution. There was an uneventful recovery in all these cases and no patient required a surgical biliary diversion.

\section{Limitations}

Our study is limited by the fact that it is a single institution observational study with no randomization. This may introduce a selection bias and further randomized controlled prospective studies are recommended. Some of the data in this study was non-normally distributed and a larger sample size is necessary to confirm our findings.

\section{Conclusion}

In patients with a large ALA, placement of a wide bore $24 \mathrm{Fr}$ catheter may be considered as an alternative to the established $10 \mathrm{Fr}$ pigtail drainage to hasten recovery of the patients. Biliary communication should be suspected in patients of ALA presenting with jaundice and placement of a biliary stent serves as a useful adjunct for their management and it may obliviate the need for a major biliary diversion surgery.

\section{Declarations}

The study was approved by the Institutional Review Board. The procedures used in this study adhere to the tenets of the Declaration of Helsinki.

\section{Ethics Approval}

This research study was conducted retrospectively from data obtained for clinical purposes. Ethical approval was granted by the Institutional Ethics Committee.

Availability of Data And Material

All patient records were obtained from the medical records department of our hospital.

Code Availability

Not applicable.

\section{Author Contributions}

All authors contributed to the study equally. J.A.G. developed the concept of study, designed the study, and wrote the manuscript. P.H.S. designed the study and wrote the 
Gandhi et al.

manuscript. S.N.C. contributed toward the study design and critical review. A.M.B. did the data collection, statistical analysis, and writing of manuscript. All authors read and approved the final manuscript.

\section{Funding \\ None.}

\section{Conflict of Interest}

None declared.

\section{Acknowledgment}

None.

\section{References}

1 van Hal SJ, Stark DJ, Fotedar R, Marriott D, Ellis JT, Harkness JL. Amoebiasis: current status in Australia. Med J Aust 2007;186(08): 412-416

2 Valenzuela O, Morán P, Gómez A, et al. Epidemiology of amoebic liver abscess in Mexico: the case of Sonora. Ann Trop Med Parasitol 2007;101(06):533-538

3 Ximénez C, Morán P, Rojas L, Valadez A, Gómez A. Reassessment of the epidemiology of amebiasis: state of the art. Infect Genet Evol 2009;9(06):1023-1032

4 SM Sandeep, Banait V, Thakur S, Bapat M, Rathi P, Abraham P. Endoscopic biliary drainage in patients with amebic liver abscess and biliary communication. Indian J Gastroenterol 2005;25(03):125-127

5 Thompson JE Jr, Forlenza S, Verma R. Amebic liver abscess: a therapeutic approach. Rev Infect Dis 1985;7(02):171-179

6 Agarwal DK, Baijal SS, Roy S, Mittal BR, Gupta R, Choudhuri G. Percutaneous catheter drainage of amebic liver abscesses with and without intrahepatic biliary communication: a comparative study. Eur J Radiol 1995;20(01):61-64

7 Rajak CL, Gupta S, Jain S, Chawla Y, Gulati M, Suri S. Percutaneous treatment of liver abscesses: needle aspiration versus catheter drainage. AJR Am J Roentgenol 1998;170(04):1035-1039

8 Saraswat VA, Agarwal DK, Baijal SS, et al. Percutaneous catheter drainage of amoebic liver abscess. Clin Radiol 1992;45(03):187-189

9 Gadahire M, Shrotriya R. A prospective comparative study to evaluate drainage of liver abscess by 20 Fr PVC catheter versus 10 Fr pigtail catheter. Ann Int Med Dent Res 2016;2(05):SG14-SG18

10 Bansal A, Bansal A, Bansal V, Kumar A. Liver abscess: catheter drainage $\mathrm{v} / \mathrm{s}$ needle aspiration. International Surgery Journal 2015;2(01):20-25

11 Singh S, Chaudhary P, Saxena N, Khandelwal S, Poddar DD, Biswal UC. Treatment of liver abscess: prospective randomized compar- ison of catheter drainage and needle aspiration. Ann Gastroenterol 2013;26(04):332-339

12 Restrepo MI, Restrepo Z, Elsa Villareal CL, Aguirre A, Restrepo M. Diagnostic tests for amoebic liver abscess: comparison of enzyme-linked immunosorbent assay (ELISA) and counterimmunoelectrophoresis (CIE). Rev Soc Bras Med Trop 1996;29(01): 27-32

13 Miller KS, Sahn SA. Chest tubes. Indications, technique, management and complications. Chest 1987;91(02):258-264

14 Cook GC. Gastroenterological emergencies in the tropics. Baillieres Clin Gastroenterol 1991;5(04):861-886

15 Christopher S, Kumar A, Shrinivas A. Liver abscess-various modalities of treatment and its clinical outcome. Int Surg J 2016;3 (04):1868-1874

16 Garale M, Takalkar Y, Ingale P. A study of the different treatment modalities used in the management of amoebic liver abscesses. Int Surg J 2016;3(04):1911-1915

17 Kumar V, Singh H. Drainage of liver abscess: comparison between $20 \mathrm{Fr}$ polyvinyl chloride versus $10 \mathrm{Fr}$ pigtail catheter. Int J Sci Stud 2017;4(11):33-36

18 Wuerz T, Kane JB, Boggild AK, et al. A review of amoebic liver abscess for clinicians in a nonendemic setting. Can J Gastroenterol 2012;26(10):729-733

19 Hughes MA, Petri WA Jr. Amebic liver abscess. Infect Dis Clin North Am 2000;14(03):565-582, viii

20 Jha AK, Jha P, Chaudhary M, et al. Evaluation of factors associated with complications in amoebic liver abscess in a predominantly toddy-drinking population: a retrospective study of 198 cases. JGH Open 2019;3(06):474-479

21 Makkar RP, Sachdev GK, Malhotra V. Alcohol consumption, hepatic iron load and the risk of amoebic liver abscess: a casecontrol study. Intern Med 2003;42(08):644-649

22 Datta DV. Amoebic liver abscess with cholestasis. J Assoc Physicians India 1977;25(12):907-912

23 Nigam P, Gupta AK, Kapoor KK, Sharan GR, Goyal BM, Joshi LD. Cholestasis in amoebic liver abscess. Gut 1985;26(02):140-145

24 Agarwal DK, Baijal SS, Roy S, Mittal BR, Gupta R, Choudhuri G. Percutaneous catheter drainage of amebic liver abscesses with and without intrahepatic biliary communication: a comparative study. 1995;20:61-64

25 Chou FF, Sheen-Chen SM, Chen YS, Chen MC. Single and multiple pyogenic liver abscesses: clinical course, etiology, and results of treatment. World J Surg 1997;21(04):384-388, discussion 388-389

26 Sharara AI, Rockey DC. Pyogenic liver abscess. Curr Treat Options Gastroenterol 2002;5(06):437-442

27 Do H, Lambiase RE, Deyoe L, Cronan JJ, Dorfman GS. Percutaneous drainage of hepatic abscesses: comparison of results in abscesses with and without intrahepatic biliary communication. AJR Am J Roentgenol 1991;157(06):1209-1212 